\title{
ISFET Glucose Sensor with Palladium Hydrogen Selective Membrane
}

\author{
Mi Kyung Chung1, Seongwan Kim¹, Sang Sik Lee², and Chong-Ook Park1,+
}

\begin{abstract}
This paper describes the development of a glucose biosensor based on ion sensitive field effect transistor(ISFET) with a palladium(Pd) modified ion sensing membrane. By adopting Pd as a hydrogen sensitive layer and integrating a screen-printed reference electrode, the sensitivity and stability were considerably improved due to the high permeability and selectivity of the Pd hydrogen selective membrane. This paper suggests a new approach for realizing portable and highly sensitive glucose sensors for diagnosing and treating diabetes mellitus.
\end{abstract}

Keywords : Glucose sensor, ISFET, Palladium, Semiconductor Biosensor

\section{INTRODUCTION}

The need to diagnose diabetes is on a constant increase as it has become one of the leading causes for death and disability around the world[1,2]. For the treatment of diabetes mellitus, maintaining a normal glucose level by monitoring and controlling the blood glucose concentration is of primary importance. This in turn can delay or prevent the occurrence of complications. Accordingly, Glucose biosensors have been extensively developed and they occupy the largest portion of the biosensor market[3]. For the purpose of glucose sensing, electrochemical type biosensors account for a large majority. They can be classified into amperometric, conductometric, potentiometric, and semiconductor biosensors.

In the recent decades, as the semiconductor market and related technologies have undergone dramatic development, semiconductor based biosensors have attracted considerable attention over other types of sensors. This is due to their advantages of small size, mass production, short response time, and facile integration with periphery processing units $[4,5]$. Ion sensitive field effect transistors(ISFETs) have been the most widely studied among the semiconductor based biosensors after Bergveld reported the first concept[6-8]. However, the use of ISFETs

${ }^{1}$ Department of Materials Science and Engineering, Korea

Advanced Institute of Science and Technology (KAIST)

${ }^{2}$ Department of Biomedical Engineering, Kwandong University

${ }^{+}$Corresponding author: cops@kaist.ac.kr

(Received: Feb. 6, 2012, Accepted : Mar. 5, 2012) for diabetes has limitations such as low sensitivity(a few $\mathrm{mV} /$ decade)[9], insufficient selectivity[10], and strong dependence on the buffer capacity[11, 12]. Palladium has been utilized as a core material for hydrogen gas sensors due to its high sensitivity and selectivity of hydrogen[13]. Hydrogen gas molecules are spontaneously dissociated on the surface and they are dissolved in palladium at room temperature. Diffused hydrogen leads to changes in the surface potential of the device. Although palladium has been used as a catalyst for the oxidation of glucose [14, 15], its high permeability to hydrogen has not been exploited until now for the detection of glucose.

This paper describes a new design for the improvement in the performance of ISFETs by adopting palladium as a hydrogen selective membrane on the sensing channel. Due to high permeability and selectivity of hydrogen in the Pd layer incorporated in the active sensing region $[13,16]$, the sensitivity and selectivity as well as the buffer capacity dependence of the glucose sensor can be enhanced. Moreover, $\mathrm{Ag} / \mathrm{AgCl}$ reference electrodes are integrated with the devices. This is achieved by using a one-step screen-printing method for miniaturization

\section{EXPERIMENT}

\subsection{Fabrication of ISFET}

Fabrication of the ISFET begins with definition of source/drain regions of p-type silicon on insulator wafer. 
Heavily n-doped source/drain regions were formed on a ptype $\mathrm{Si}$ layer $(10 \Omega \cdot \mathrm{cm})$ with a spin-on-dopant at $950{ }^{\circ} \mathrm{C}$ in a rapid thermal annealing system. A photolithographically defined layer of $\mathrm{SiO}_{2}$, deposited by plasma enhanced chemical vapor deposition and it was used as a diffusion mask. After doping process, the active regions of the transistors were isolated by $\mathrm{SF}_{6}$ plasma etching. Ion sensitive layers of silicon oxide $\left(\mathrm{SiO}_{2}, 90 \mathrm{~nm}\right)$ and silicon nitride $\left(\mathrm{Si}_{3} \mathrm{~N}_{4}, 110 \mathrm{~nm}\right)$ were deposited by plasma enhanced chemical vapor deposition, respectively. Palladium was deposited on top of the $\mathrm{Si}_{3} \mathrm{~N}_{4}$ layer by $\mathrm{RF}$ sputtering. The deposited Pd of $50 \mathrm{~nm}$ thickness was patterned by using a lift-off process. After patterning the source and drain electrodes of $\mathrm{Cr} / \mathrm{Au}(10 \mathrm{~nm} / 200 \mathrm{~nm})$ by using standard lithography, the device was passivated by spin-cast SU-8 thin layers except for the channel region and metal pads.

\subsection{Integration of Screen-Printed Reference Electrode}

The $\mathrm{Ag} / \mathrm{AgCl}$ reference electrode was fabricated by using a screen-printing technique. Initially, a silver paste(DS-0715AT(N) DAEJOO Electronic Materials Co. Ltd.) was printed onto the substrate of the device through a screen of mesh size 250 and dried at $120^{\circ} \mathrm{C}$ for $10 \mathrm{~min}$. After the formation of an $\mathrm{Ag}$ metal line, $\mathrm{Ag} / \mathrm{AgCl}$ ink(SC155 Screen-Printable $\mathrm{Ag} / \mathrm{AgCl}$ paste, KETI) was printed onto the device by using a 250 mesh screenprinting mask(12 $\mu \mathrm{m}$ thickness $)$. The device was then treated at $120{ }^{\circ} \mathrm{C}$ for $10 \mathrm{~min}$ and cleaned with deionized water.

\subsection{Immobilization of Enzyme on the Ion Sensitive Layer}

In order to immobilize the glucose oxidase $(\mathrm{GOx})$ (EC.1.1.3.4, Sigma Aldrich) enzyme on the substrate, $5 \mathrm{mg}$ of GOx and $5 \mathrm{mg}$ of bovine serum albumin was dissolved in a phosphate buffer solution(PBS). In order to establish cross linking, a GOx solution and $2.5 \%$ of glutaraldehyde (GA) were mixed at a ratio of 10:1 and dropped on the gate surface. After dropping $5 \mu \mathrm{L}$ of the mixed enzyme solution, the surface was dried at room temperature for $6 \mathrm{~h}$ and washed with deionized(DI) water to remove unattached GOx.

\section{RESULTS AND DISCUSSION}

Fig. 1 shows the schematic representation of the device structure of the ISFET glucose sensor with an additional Pd hydrogen selective membrane. The upper inset of Fig. 1 shows a cross-sectional scanning electron microscopy (SEM) image of uniform ion selective gate layers. The specific enzyme glucose oxidase was immobilized on the Pd surface for the detection of glucose[11]. The overall reaction of glucose oxidase between glucose and dioxygen can be written in the form of the following equations.

$$
\begin{aligned}
& \beta \text { - D - Glucose }+\mathrm{O}_{2} \rightarrow \mathrm{D} \text { - glucono }-\delta \text { - lactone } \\
& \text { (or gluconic acid) }+\mathrm{H}_{2} \mathrm{O}_{2} \\
& \text { D - glucono - } \delta \text { - lactone (or gluconic acid) } \\
& \leftrightarrow \text { D-gluconate }+\mathrm{H}+
\end{aligned}
$$

Glucose is oxidazed into gluconolactone by glucose oxidase while the reduction of dioxygen by GOx yields hydrogen peroxide. Gluconolactone, or gluconic acid in the hydrolysed form, dissociates and releases $\mathrm{H}+$ ions which is is the target of glucose detection using ISFET.

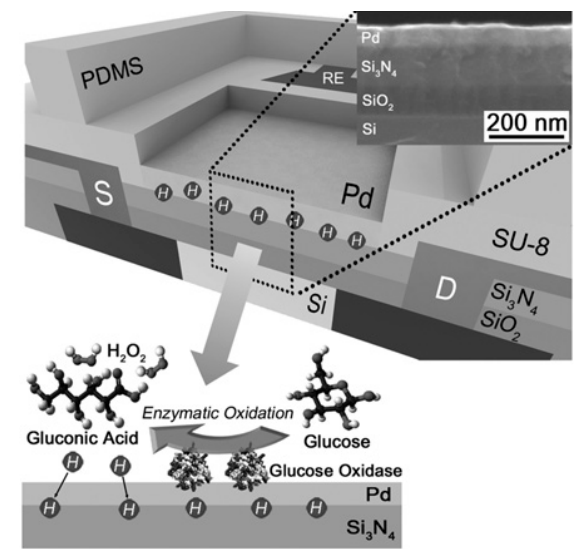

Fig. 1. A schematic of the device structure of the ISFET glucose sensor with an additional Pd hydrogen selective membrane on the sensing channel.

Fig. 2a shows the output performance characteristics of ISFET without a Pd layer immersed in $\mathrm{PBS}(\mathrm{pH} 7)$ solution. The gate voltage $\left(\mathrm{V}_{\mathrm{G}}\right)$ was applied through the reference electrode from $-2 \mathrm{~V}$ to $3 \mathrm{~V}$. The drain current $\left(\mathrm{I}_{\mathrm{D}}\right)$ was plotted as a function of the drain voltage $\left(\mathrm{V}_{\mathrm{G}}\right)$ swept from $0 \mathrm{~V}$ to $3 \mathrm{~V}$ while $\mathrm{V}_{\mathrm{G}}$ is applied. This device has a threshold voltage $\left(\mathrm{V}_{\mathrm{T}}\right)$ of $0.2 \mathrm{~V}$ with an on/off ratio of 104 obtained from the $I_{D}-V_{G}$ curve(transfer curve) at $V_{D}=1 \mathrm{~V}$. 
This is shown in the inset of Fig. 2a. The transistor device showed stable performance and it worked consistently with the screen-printed $\mathrm{Ag} / \mathrm{AgCl}$ reference electrode as a gate.

Fig. $2 b$ shows the $I_{D}-V_{G}$ characteristics of the ISFET without the Pd layer in different $\mathrm{pH}$ solutions at $\mathrm{V}_{\mathrm{D}}=1 \mathrm{~V}$. Instead of varying $\mathrm{V}_{\mathrm{G}}$, the $\mathrm{pH}$ of the standard solutions in which the ISFET was immersed was changed from $\mathrm{pH} 10$ to 4. At a constant $\mathrm{V}_{\mathrm{D}}, \mathrm{V}_{\mathrm{T}}$ decreased $(51.7 \mathrm{mV} / \mathrm{pH})$ as the concentration of $\mathrm{H}^{+}$ion increased. These results indicate that the threshold voltage of the transistor is decreased, as an increased amount of positive ions $\left(\mathrm{H}^{+}\right)$attract more electrons in the silicon channel region[11]. This result coincides with the conventional $\mathrm{pH}$ sensitivity of $\mathrm{Si}_{3} \mathrm{~N}_{4}$, which is known to have $46 \mathrm{mV} / \mathrm{pH} \sim 56 \mathrm{mV} / \mathrm{pH}$ (Matsuo et al., 1974).

(a)

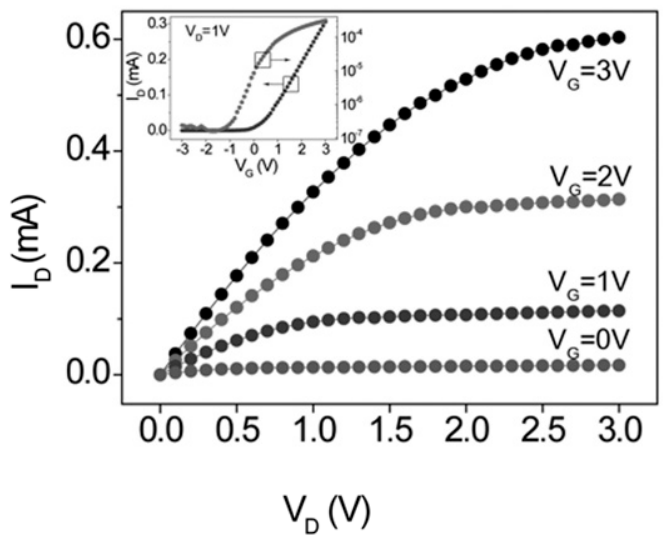

(b)

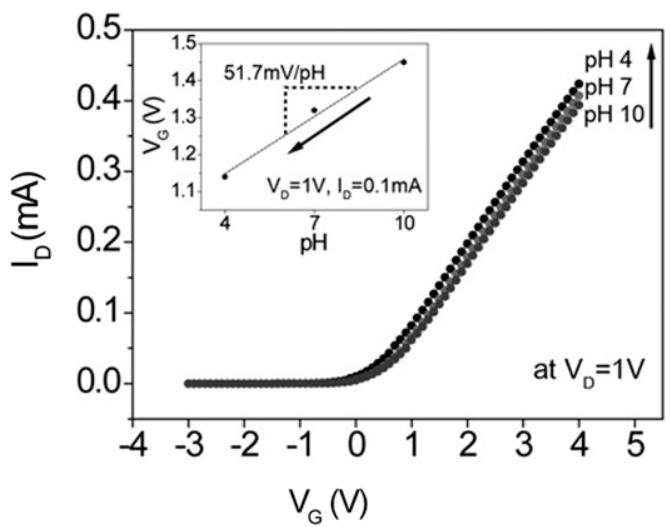

Fig. 2. (a) Output characteristics of the ISFET without a Pd layer immersed in PBS ( $\mathrm{pH} 7$ ) solution. (b) $\mathrm{I}_{\mathrm{D}}-\mathrm{V}_{\mathrm{G}}$ characteristics of the ISFET without the Pd layer in different $\mathrm{pH}$ solutions at $\mathrm{V}_{\mathrm{D}}=1 \mathrm{~V}$.

Fig. 3a shows the schematic illustration of the hydrogen ion detection mechanism of the ISFET with a Pd hydrogen selective membrane. Hydrogen is known to be adsorbed at the outer surface of $\mathrm{Pd}$, and then diffuses through the Pd layer[13, 17]. The produced hydrogen ions are converted to a hydrogen atom by accepting an electron from $\mathrm{Pd}$. These hydrogen ions diffuse through the Pd layer and they combine with the binding sites on the surface of $\mathrm{Si}_{3} \mathrm{~N}_{4}$. At the Pd-insulator interface, the bounded hydrogen changes the work function of the interface by forming a dipole[17]. Thus, the threshold voltage of the transistor changes as follows:

$$
\Delta \mathrm{V}_{\mathrm{T}}=-\mu \mathrm{N} \theta / \square_{0}
$$

where, $\mu$ is the dipole moment of hydrogen, $\mathrm{N}$ is the density of adsorption sites, $\theta$ is the fractional coverage of adsorbed hydrogen atoms, and $\square_{0}$ is the dielectric permittivity in the free space. In this case, the fractional coverage depends on the $\mathrm{H}^{+}$ion concentration.

$$
\theta /(1-\theta)=\text { const }\left[\mathrm{H}^{+}\right]
$$

More hydrogen atoms are located at the $\mathrm{Pd} / \mathrm{Si}_{3} \mathrm{~N}_{4}$ interface compared to the solution-nitride interface without $\mathrm{Pd}$. As a result, more reactions between hydrogen ions and $\mathrm{SiNH}_{2}$ can occur. Therefore, potential changes become greater when the Pd layer exists due to the increased fractional coverage.

Comparison of the glucose response of the ISFET glucose sensor without and with an additional Pd layer is shown in Fig. 3b. The drain current of the ISFET with the Pd layer increased sharply compared to the current of the ISFET without the $\mathrm{Pd}$ layer. At $9 \mathrm{mM}$ glucose concentration, $\Delta \mathrm{I}_{\mathrm{D}}$ increased from $11 \mu \mathrm{A}$ to $32 \mu \mathrm{A}$ with the adoption of the Pd layer. As depicted in Figure $3 \mathrm{a}$, the hydrogen adsorption property and the permeability of $\mathrm{Pd}$ can improve the selectivity and lessen the interference effects of other ions in the electrolyte solution[18]. Fig. 3c presents the comparison between the gate voltage changes of the ISFET without and with the Pd layer. At high glucose concentration, the rate of increase of the drain current decreased. This is due to the limited dynamic range of the ISFET which is caused by the oxygen limitation via biocatalytic glucose oxidation[19]. The ISFET with the Pd layer showed glucose sensitivity of roughly $16.48 \mathrm{mV} / \mathrm{mM}$ with a $0.96 \% \mathrm{r}^{2}$ while the ISFET without the Pd layer presented $7.98 \mathrm{mV} / \mathrm{mM}$ glucose sensitivity. As seen from the results, the glucose sensitivity increased twofold by applying an additional Pd layer and this causes an increase in the amount of hydrogen ions which are transported to the $\mathrm{Si}_{3} \mathrm{~N}_{4}$ surface. The methods described in this work may 
offer a solution for the realization of a highly sensitive glucose biosensor.
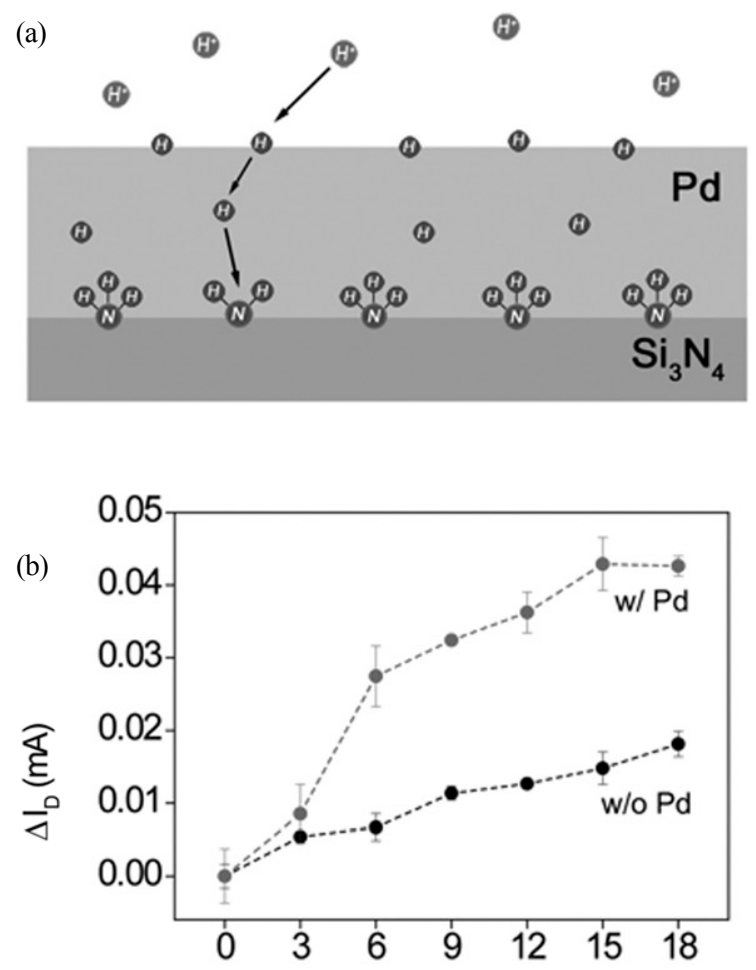

Glucose Concentration (mM)

(c)

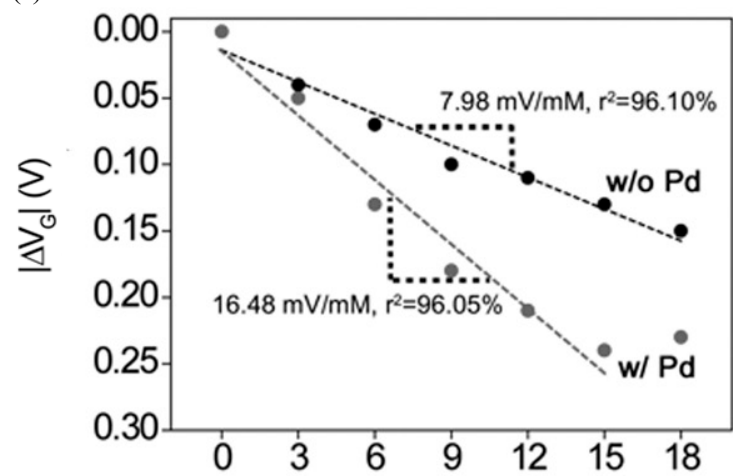

\section{Glucose Concentration (mM)}

Fig. 3. (a) Schematic illustration of the hydrogen ion detection mechanism of the ISFET with a Pd hydrogen selective layer. (b) Comparison of the glucose response of the ISFET glucose biosensor without and with an additional Pd layer. (c) Comparison between the gate voltage changes of the ISFET without and with the Pd layer.

\section{CONCLUSION}

We developed an ISFET glucose biosensor with an additional Pd layer. A palladium thin layer was adopted in an ion selective gate membrane in order to increase the hydrogen adsorption property and permeability to the silicon nitride surface. The ISFET glucose sensor with an additional Pd layer showed increased sensitivity and selectivity compared to an ISFET glucose sensor without any additional layer. Moreover, one step fabrication of a screen-printed $\mathrm{Ag} / \mathrm{AgCl}$ reference electrode can be applied to the compact bio-sensing device. This ISFET with the Pd layer is expected to provide a new opportunity for the practical application of semiconductor based glucose biosensors.

\section{ACKNOWLEDGMENT}

This work was supported by the Korean Ministry of Environment under the "Converging technology project".

\section{REFERENCES}

[1] K.M.V. Narayan, J.B. Saaddine, J.P. Boyle, T.J. Thompson, and L.S. Geiss, "Impact of recent increase in incidence on future diabetes burden", Diabetes Care, vol. 29, no. 9, pp. 2114-2116, 2006.

[2] E.H. Yoo and S.Y. Lee, "Glucose biosensors: an overview of use in clinical practice", Sensors, vol. 10, no. 5, pp. 4558-4576. 2010.

[3] A. Heller and B. Feldman, "Electrochemical glucose sensors and their applications in diabetes management", Chem. Rev., vol. 108, no. 7, pp. 2482-2505, 2008.

[4] S.V. Dzyadevych, A.P. Soldatkin, Y.I. Korpan, V.N. Arkhypova, A.V. El'skaya, J.M. Chovelon, C. Martelet, and N. Jaffrezic-Renault, "Biosensors based on enzyme field-effect transistors for determination of some substrates and inhibitors", Anal. Bioanal.Chem., vol. 377, no. 3, pp. 496-506, 2003.

[5] N. Jaffrezic-Renault and C. Martelet, "Semiconductorbased micro-biosensors", Synth. Met., vol. 90, no. 3, pp. 205-210, 1997.

[6] P. Bergveld, "Development of an ion-sensitive solidstate device for neurophysiological measurements", IEEE Trans. Biomed. Eng., vol. BME-17, no. 1, pp. 7071, 1970.

[7] P. Bergveld, "Development, operation, and application of the ion-sensitive field-effect transistor as a tool for electrophysiology", IEEE Trans. Biomed. Eng., vol. BME-19, no. 5, pp. 342-351, 1972. 
[8] M.W. Shinwari, M.J. Deen, and D. Landheer, "Study of the electrolyte-insulator-semiconductor field-effect transistor(EISFET) with applications in biosensor design", Microelectron. Reliab., vol. 47, no.12, pp. 2025-2057, 2007.

[9] A.A. Shulga, A.C. Sandrovsky, V.I. Strikha, A.P. Soldatkin, N.F. Starodub, and A.V. Elskaya, "Overall characterization of ISFET-based glucose biosensor", Sens. Actuators B, vol. 10, no. 1, pp. 41-46, 1992.

[10] A.K. Covington and P.D. Whalley, "Recent advances in microelectronic ion-sensitive devices(ISFETs)", $J$. Chem. Soc., Faraday Trans. 1, vol. 82, pp. 1209-1215, 1986.

[11] S.V. Dzyadevych, A.P. Soldatkin, A.V. El'skaya, C. Martelet, and N. Jaffrezic-Renault, "Enzyme biosensors based on ion-selective field-effect transistors", Anal. Chim. Acta, vol. 568, no. 1-2, pp. 248-258, 2006.

[12] M.J. Schoning and A. Poghossian, "Recent advances in biologically sensitive field-effect transistors (BioFETs)", Analyst, vol. 127, no. 9, pp. 1137-1151, 2002.

[13] I. Lundstrom, C. Nylander, and A. Spetz, "Palladiumsilicondioxide-silicon structures as hydrogen sensors in electrolytes", Electron. Lett., vol. 19, no.7, pp. 249$251,1983$.

[14] Q.J. Chi and S.J. Dong, "Flow-injection analysis of glucose at an amperometric glucose sensor based on electrochemical deposition of palladium and glucose oxidase on a glassy carbon electrode", Anal. Chim. Acta, vol.278, no.1, pp. 17-23, 1993.

[15] S.H. Lim, J. Wei, J.Y. Lin, Q.T. Li, and J. KuaYou, "A glucose biosensor based on electrodeposition of palladium nanoparticles and glucose oxidase onto Nafion-solubilized carbon nanotube electrode", Biosens. Bioelectron., vol. 20, no. 11, pp. 2341-2346, 2005.

[16] Z. Shi and J.A. Szpunar, "Synthesis of an ultra-thin palladium membrane for hydrogen extraction", Rev. Adv. Mater. Sci., vol. 15, no.1, pp. 1-9, 2007.

[17] K.I. Lundstrom, M.S. Shivaraman, and C.M. Svensson, "A hydrogen-sensitive Pd-gate MOS transistor", J. Appl. Phys., vol. 46, no. 9, pp. 38763881, 1975.

[18] R. Kirchheim and R.B. McLellan, "Electrochemical methods for measuring diffusivities of hydrogen in palladium and palladium alloys", J. Electrochem. Soc., vol. 127, no. 11, pp. 2419-2425, 1980.

[19] V. Volotovsky, A.P. Soldatkin, A.A. Shulga, V.K. Rossokhaty, V.I. Strikha, and A.V. Elskaya, "Glucose-sensitive ion-sensitive field-effect transistorbased biosensor with additional positively charged membrane Dynamic range extension and reduction of buffer concentration influence on the sensor response", Anal. Chim. Acta, vol. 322, no. 1-2, pp. $77-$ 81, 1996.

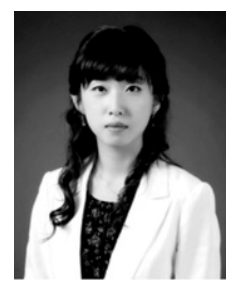

Mi Kyung Chung received her B.S. degree(2010) and M.S. degree(2012) in department of materials science and engineering from KAIST. Now, she is a researcher in LG Chem. Ltd.

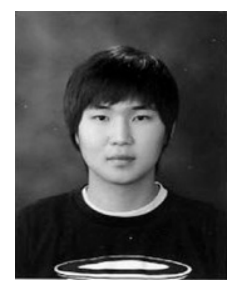

Seong Wan Kim received his B.S. degree(2010) and M.S. degree(2011) in department of materials science and engineering from Korea Advanced Institute of Science and Technology. Now, he is a doctoral course student of the Department of Material Science and Engineering in Korea Advance Institute Science and Technology. 




Sang Sik Lee received the B.S., M.S. and Ph.D. degrees in Department of Biomechatronic Engineering, all from Sungkyunkwan University, Suwon, Korea in 1993, 1996 and 2000, respectively. From 1993 to 2000, he worked at LG Cable Co.Ltd. R\&D Complex as a team manager. From 2001 to 2004, he worked at Mido Co. Ltd. as an executive. From 2004 to 2006 he was a research associate professor in Biomedical Engineering Lab. of Sung kyunkwan University, Suwon, Korea. Since 2011, he has been a professor in Department of Biomedical Engineering, Kwandong University, Gangneung, Korea.

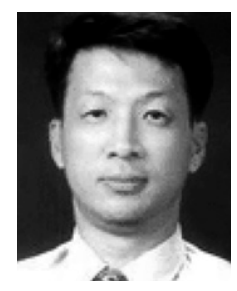

Chong-Ook Park received the B.S. degree(1979) in the metallurgical engineering from the Seoul National University and the Ph.D. degree(1985) in the materials science and engineering from the Ohio State University. From 1985 to 1986, he was with University of Pennsylvania as a Post Doc and worked at Goldstar central Research Lab. as the director from 1986 to 1988. Since 1990, he has been a technical adviser at LG Corporate Institute of Technology. He joined the department of materials science and engineering at the Korea Advanced Institute of Science and Technology as an assistant professor in 1988 and he has been a professor since 1999. His current research fields are gas sensors, especially electrochemical devices. 